Original Article

\title{
COM PARATIVE STUDY ON THE EFFECTIVENESS OF LOW LEVEL LASER THERAPY VERSUS PHONOPHORESIS IN THE MANAGEMENT OF LATERAL EPICONDYLITIS
}

\author{
Mohamed Faisal C.K. ${ }^{1}$, Mary Sumila ${ }^{2}$, Lawrence Mathias ${ }^{3} \&$ Ajith S. \\ ${ }^{1}$ Professor \& Vice Principal, ${ }^{2}$ Physiotherapist, ${ }^{4}$ Assistant Professor, \\ Nitte Institute of Physiotherapy, Nitte University, M angalore - 575 018, India. \\ ${ }^{3}$ Professor, Department of Orthopaedics, K.S. Hegde Medical Academy, \\ Nitte University, M angalore - 575 018, India.
}

Correspondence:

Mohamed Faisal C.K.

Professor \& Vice Principal, Nitte Institute of Physiotherapy, Nitte University, M angalore - 575 018, India. E-mail : faisal77mpt@gmail.com

\begin{abstract}
:
Background and Objectives: The use of electro physical agents for the management of any soft tissue injuries in Physical Therapy practice is very common for an early recovery from the injury. Among that, Low Level Laser Therapy (LLLT) and Phonophoresis are found to be very effective in soft tissue injuries. So the objective of this study is to know the comparative effectiveness of LLLT versus Phonophoresis in the treatment of lateral epicondylitis.

M ethods: Convenient sample of 40 patients with lateral epicondylitis were recruited for this study. They were assigned equally either to a laser $(n=20)$ or a Phonophoresis $(n=20)$ group. Laser group received the treatment with Ga-As $(904 n m)$ IR semiconductor diode laser. For Phonophoresis group a gel containing $1 \%$ sodium diclofenac was used as coupling agent. Each group was treated 3 times a week, for a total of 8 treatments, and was evaluated subjectively and objectively before and at the end of the treatment, by using VAS, pain free grip strength and functional pain scale as variables.

Results: Comparison between laser group and Phonophoresis group is done using $\mathrm{M}$ ann Whitney $\mathrm{U}$ test. Comparison between pre and post is done using Wilcoxon's signed rank sum test. Form selection to the post treatment assessment, a significant decrease in symptoms was found in both laser and phonophoresis groups. But it is concluded that statistically; low level laser therapy was not significantly better than phonophoresis with the results of VAS $(P=0.53), \operatorname{FPS}(P=0.253)$, pain free grip strength. $(P=0.426)$.

Interpretation and conclusions: The result of this study suggest that low level laser therapy as well as phonophoresis with sodium diclofenac gel as couplant medium gives a good result in the management of lateral epicondylitis. This study concludes that statistically there was no significant difference between Low level laser therapy and Phonophoresis with sodium diclofenac gel as coupling agent in the management of lateral epicondylitis.
\end{abstract}

Keywords : Lateral epicondylitis; Low level laser Therapy, Phonophoresis, VAS, Pain free Grip Strength, Functional Pain Scale.

\section{Introduction:}

Tennis elbow or lateral epicondylitis, is generally a work related or sport related pain disorder of the common extensor origin of the arm, Access this article online Quick Response Code

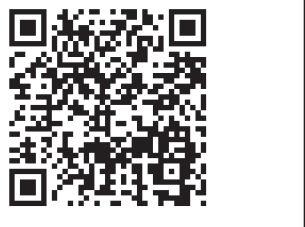
usually caused by excessive quick, repetitive movements of the wrist and forearm ${ }^{1}$. Tendon injuries about the elbow are usually the result of overuse from repetitive tension overload. An overuse injury occurs when tissue damage occurs over time at a rate exceeding the body's ability to heal it. As the stress tolerance of the tendon is exceeded, inflammation or tissue breakdown may occur, leading to pain or disability. The patient does not have to be a competitive athlete to develop overuse injuries about the elbow ${ }^{2}$. A tennis elbow may be provoked by any exercise involving repeated and forcible extension movements at the wrist-not necessarily tennis-and bears its name only because, it was first described as a tennis player's disability ${ }^{3}$. In clinical practice the condition is found more 
often in non-athletes than athletes; probably less than $5 \%$ of patients with epicondylitis are golf or tennis players ${ }^{4}$. Musicians, Carpenters, Assembly-line workers and many others subject themselves to repetitive activities on an almost daily basis, which may lead to overuse. Work or play that requires pronation and supination, overuse of the wrist or finger extensors, or lifting with the hands in the palm down position are believed to be predisposing factors ${ }^{2}$. On the whole, English writer have called it 'tennis elbow' while continental authors have preferred 'Epicondylitis's.

Tennis elbow is more frequent in patients over 35 years but may occur at a younger age in highly competitive athletes who subject their tendons to greater physiological demands. The peak age of onset is between 40 and 50 years, averaging 22.6 years of prior tennis experience ${ }^{2}$. The dominant arm is affected in most cases and the condition is bilateral in a few. Men are more likely to suffer than women ${ }^{4}$. Extreme moments of force or repetition, despite reasonable muscle power, endurance and flexibility can lead to fatigue and subsequent failure. Overuse is encountered when the body's physiological ability to heal lags behind the micro trauma occurring with the repetitive action $^{5}$. The onset of symptoms is usually gradual, often appearing after vigorous activity. Less commonly, acute onset may be associated with an extreme effort or direct trauma. In many instances no predisposing activity can be determined ${ }^{2}$.

In most cases the lesion involves the specialised junctional tissue at the origin of the common extensor muscle at the lateral humeral epicondyle, specifically the tendinous origin of extensor carpi radialis brevis. The lesion is characterised by macroscopic and microscopic tears, which may be superficial or deep and situated at the tendinous origin of the extensor carpi radialis brevis into the periosteum of the lateral humeral epicondyle 4 . In addition, the anterior edge of the origin of the extensor digitorum communis is involved in $30 \%$ of cases. Rarely, the underside of the Extensor Carpi Radialis Longus or the origin of the Extensor Carpi Ulnaris is involved ${ }^{2}$. In most patients aged over 30 the condition may be considered to be a degenerative process, the onset of symptoms being hastened by overuse of the $\mathrm{arm}^{4}$. Initial inflammatory changes may produce a characteristic tendinitis, but as the chronic condition develops, the degenerative features of tendinosis are thought to become paramount. The sequence of degeneration included changes in the blood vessel walls and fibroblasts and alteration in the gel-tofibre ratio, with glycosaminoglycans replacing collagen fibres. This led to lack of maintenance of collagen turnover, loss of the wavy configuration of collagen fibres and transformation of the fibroblasts into chondrocyte-type cells, with subsequent cartilage formation, calcification and eventual bone formation ${ }^{6}$.

Treatment is aimed at relief of inflammation, promotion of healing, reducing the overload forces, and increasing upper extremity strength, endurance and flexibility. Physiotherapy treatment initially consists of assessment, modification of activity, and application of ice and selected modalities. Electrotherapy modalities such as laser, ultrasound, phonophoresis with 10 percent hydrocortisone, interferential therapy, high voltage galvanic stimulation, and transcutaneous electrical nerve stimulation have all been advocated to relieve pain and inflammation. Cure rates have been reported from 55 to 90 percent. Manual therapy techniques, e.g., transverse frictions, joint mobilization and manipulation, myofascial release, and strain and counter strain techniques may also be used. Whatever the treatment employed, as resolution occurs and the patient returns to the sport or occupation, exercise is a mainstay of the treatment. Complete rest is seldom indicated ${ }^{7}$.

Laser energy emitted from the active medium as photons focused on the skin via a laser probe, is postulated to be absorbed essentially by photosensitive molecules called chromophores. There is a consensus that low level laser therapy (LLLT) induces, on biological tissues, a photobiomodulation effect. This photobiomodulation effect implies manifestation of photochemical reactions as opposed to photothermal reactions, such as those 
observed with other therapeutic lights. This phenomenon of photobiomodulation has been applied clinically in the treatment of soft tissue injuries ${ }^{8}$.

Phonophoresis is the movement of drugs through skin into the subcutaneous tissues under the influence of ultrasound (US). M any drugs are absorbed through the skin very slowly; high-frequency sonic vibration may accelerate this process ${ }^{9}$. Both the thermal and non thermal (mechanical) properties of ultrasound have been cited as possible mechanisms for the transdermal penetration of the pharmacological agents ${ }^{10}$. Gastrointestinal upset and local pain commonly limits the use of oral nonsteroidal anti-inflammatory drugs and corticosteroid injection as treatments for lateral epicondylitis. Transdermal administration of an anti-inflammatory drug could avoid these adverse effects. Topical 2\% diclofenac in Pluronic lecithin liposomal organo-gel appears to provide effective short-term reduction in elbow pain and wrist extensor weakness associated with chronic lateral epicondylitis ${ }^{11}$. Therapeutic ultrasound irradiation enhances the percutaneous penetration of to pical diclofenac ge ${ }^{12}$.

Pain, strength, and functional status are the outcomes most commonly monitored in lateral epicondylitis clinical trials. A $10 \mathrm{~cm}$ visual analogue scale (VAS) has frequently been used to measure pain intensity. The VAS is considered responsive because changes in pain VAS scores were greater for patients successfully responding to treatment than the scores for those who failed to respond to treatment. Strength measures used in clinical trials for lateral epicondyle include pain-free grip, maximum grip and isokinetic torque of the forearm and wrist. When compared with maximum grip, pain-free grip appears to be more suitable for clinical trials of lateral epicondyle because it has stronger correlations with pain and function and is more responsive. The Functional pain scale (FPS) is an easily administered, reliable, valid and responsive measure of functional status suitable for evaluating changes in pain-related elbow function in subjects with lateral epicondylitis ${ }^{13}$.

Even though Laser therapy and phonophoresis are two effective treatments for lateral epicondylitis, ${ }^{12,}$ 14, 15,16,17,18 there is scant data available regarding the relative efficacy of low level laser therapy versus phonophoresis in the management of lateral epicondylitis .So this study is designed to compare the relative efficacy of low level laser therapy versus phonophoresis by using pain, pain free grip strength, functional pain scale as variables which are proven for their usefulness for this study from the point view of reliability and construct validity.

\section{Objectives:}

To compare the effectiveness of Low level laser (Gallium -Arsenide) versus Phonophoresis in patients with lateral epicondylitis, by using pain, pain free grip strength, and functional pain scale as evaluative variables.

\section{Materials \& Methodology:}

A total of 40 subjects, both males and females, in age group of 21 -51 years suffering from lateral epicondylitis were referred from outpatient department of K.S. Hegde Charitable Hospital, Mangalore and by private Orthopaedicians who were diagnosed by the Orthopaedicican as having Tennis Elbow were selected for the study. All patients with lateral elbow pain were assessed and those who fulfilled the inclusion criteria alone were selected and assigned to any of the two treatment groups. Group A $(n=20)$ were given phonophoresis therapy and group $B(n=20)$ were given low level laser therapy. This study was approved by the Institutional Ethical Committee of Nitte Institute of Physiotherapy and a written consent was taken from each patient before the study. The inclusion criteria ${ }^{19}$

were; those who were having typical history of localized pain and tenderness at the antero-inferior aspect of lateral epicondyle aggravated by gripping and wrist extension activities and relieved by rest, Positive Cozens test, Positive Millstest.

The exclusion criteria were; Cervical spondylosis, associated with radiating pain on lateral epicondyle, any neurological conditions, Previous fracture humerus, olecranon, radius or ulna, Rheumatoid arthritis, Patients 
injected with corticosteroids in preceding 6 months, Radio humeral bursitis,

Each patient was evaluated subjectively and objectively before starting the treatment and at the end of eight sessions of treatment. The outcome tools used were; Visual analogue scale (VAS), Functional pain scale and Pain free grip strength by hand dynamometer.

The pain free grip strength was measured by making the subject stood holding the grip dynamometer with the elbow extended and forearm in neutral position. To test pain free grip strength the subjects squeezed the dynamometer with the involved limb until they first experience pain. Pain-free grip was calculated by averaging the values of three consecutive repetitions separated by a 20 -second rest (Figure 1 ).

\section{LaserTreatment Methodology:}

The nature of the treatment and the need to wear goggles are explained to the patient and also the patients were instructed about the time of application and duration of treatment. The laser apparatus is conveniently positioned. Protective goggles, designed for the particular wavelength being used, are worn to obviate any risk of accidental application of the laser beam into the eye. The part was supported in such a way that any pressure of the laser applicator does not cause movement or discomfort. The patient was made to sit in the chair with the involved arm abducted and elbow flexed resting on a pillow.

A hand held applicator which is continuous semiconductor $\mathrm{Ga}$ As laser of $904 \mathrm{~nm}$ wavelength was placed on the treatment points, marked on the patient's skin so as to make right angle with the skin and the beam is directed into the patient at that point. The laser applicator is applied to the surface before switching on. A firm contact should be maintained throughout treatment but should not provoke pain where tenderness is present. Direct application to the skin ensures maximum transfer of laser energy and light pressure; squeezing blood from superficial vessels can increase the penetration further. The position is maintained for necessary time. The device is switched off before removing the applicator from skin contact. (Figure 2)

\section{Specifications:}

Name of the laser unit : IR27Laser Ronald Series unit.

Lasertype

: Semi conductor Gallium Arsenide Laser.

Laserwavelength : $904 \mathrm{~nm}$

Frequency : : $10 \mathrm{~Hz}$.

Output Power : $: 0.096 \mathrm{mw}$.

Density of energy $\quad: 3.5 \mathrm{~J} / \mathrm{cm}^{2}$.

Divergency

Duration

: $16.5710^{-3} \mathrm{~cm}^{2}$.

Treatment protocol

: 10 minutes.

: Thrice weekly for total 8 treatments sessions ${ }^{14}$.

\section{Phonophoresis Methodology :}

The nature of the treatment, need for a couplant and stability of the area are all explained to the patient. The patient was made to sit in the chair with the involved arm abducted and elbow flexed resting on a pillow. The couplant should be applied to the skin surface. Sodium diclofenac was used as the coupling medium. The patients were asked to keep the part to be treated still and relaxed and to report any increase of pain or other sensations immediately. The treatment head was placed on the skin before the output is turned on. This is to avoid damage to the transducer which can occur if the energy is reflected back into the transducer at its interface with air. The treatment head is moved continuously over the surface while even pressure is maintained in order to iron out the irregularities in the sonic field. The emitting surface must be kept parallel to the skin surface to reduce reflection and pressed sufficiently firmly to exclude any air. The rate of movement must be slow enough to allow the tissues to deform and thus remain in complete contact with rigid treatment head but fast enough to prevent 'hot spots' developing when using a higher-intensity treatment. The intensity was returned to zero, before the transducer is removed from the tissue contact (Figure 3). 
Dosimetry:

Machine

: Intellect Mobile Combo (Model

2778) manufactured by

Chattanooga, M exico

$1 \%$ sodium Diclofenac is used as coupling medium.

$\begin{array}{ll}\text { Frequency } & : 1 \mathrm{M} \mathrm{Hz} \\ \text { Mode } & : \text { Continuousmode } \\ \text { Intensity } & : 2 \mathrm{~W} / \mathrm{cm}^{2} \\ \text { Duration } & : 7 \mathrm{M} \text { inutes }\end{array}$

Treatment protocol : 3 times weekly for 8 treatment sessions $^{17,20,21}$.

\section{Results:}

The data were analyzed by using Wilcoxon signed rank sum test for the intra group comparison and $M$ ann-Whitney $U$ test for the inter group comparison to know the improvement in hand function of Control and Experimental groups. Statistical analysis was performed with the SPSS Vers. 10.0program. A .05\% of probability was adopted as the level for statistical significance.

Table 1 shows mean value for age in group $A$ as 34.9 6.6879 and group $B$ as 33.8 10.1442. There is no significant difference $(P=0.849)$ between group $A$ and group $B$. Both groups were similar with respect to age.

Table 2 shows, both sexes were included in the study among which total numbers of males were $23(57.5 \%)$ and females were 17 (42.5\%). Group A includes 12 males (60\%) and 8 females (40\%). Group B includes 11 males (55\%) and 9 females (45\%). Statistical analysis shows no significant difference $(P=0.749$ ) between group $A$ and group $B$ in male to female ratio.

Table 3 shows equal number of dominant (85\%) and non dominant hand (15\%) was involved in both the groups $A$ and $B$. Analysis shows both groups were similar with respect to dominant to non dominant hand ratio.

Table 4 shows, Pretreatment mean value of VAS for group A is 5.9 1.4614 and group B is 5.7 1.1098. Statistical analysis shows no significant difference $(P=0.435)$ between the 2 groups on VASbefore the treatment.
Pretreatment mean value of FPS for group A is 23.63 .9523 and group $B$ is 24.6 3.6332. Statistical analysis shows no significant difference $(P=0.414)$ between the 2 groups on FPS before the treatment.

Pretreatmentmean value of pain freegrip strength for group A is $13.95 \quad 5.3653$ and group B is 14.43 .5748 . Statistical analysis shows no significant difference $(P=$ 0.353 ) between the 2 groups on pain free grip strength before the treatment.

Table 5 shows, Paired difference of pre to post scores on VASfor Group A gives a mean value of 3.351 .9042 which is statistically $(P=0.001)$ very highly significant. Result indicates on the basis of VAS that, there is good improvement following treatment.

Paired difference of pre to post scores on FPS for Group A gives a mean value of 11.67 .1259 which is statistically $(P=$ 0.001 ) very highly significant. Result indicates on the basis of FPS that, there is good improvement following treatment.

Paired difference of pre to post scores on pain free grip strength for Group A gives a mean value of -5.55 4.6957 which is statistically $(P=0.001)$ very highly significant. Result indicates that, there is good improvement for pain free grip strength following treatment.

Paired difference of pre to post scores on VAS for Group B gives a mean value of 3.11 .4510 which is statistically $(P=$ .001 ) very highly significant. Result indicates on the basis of VAS that, there is good improvement following treatment.

Paired differences of pre to post scores on FPS for group $B$ gives a mean value of 8.353 .5285 which is statistically $(P=$ 0.001 ) very highly significant. Result indicates on the basis of FPS that there is good improvement following treatment.

Paired differences of pre to post scores on pain free grip strength For group B gives a mean value of $-4.7 \quad 3.4504$ which is statistically $(P=0.001)$ very highly significant. Result indicates that there is good improvement for pain 
free grip strength following treatment. (Graphs 1-3)

Table 6 shows group statistic analysis for VAS, $(P=0.53)$ FPS $(P=0.253)$ and $P$ ain free grip strength $(P=0.426)$ shows that statistically there is no significant difference in the comparative effectiveness of both treatment modalities.

\section{Discussion :}

This study has shown that low level laser therapy as well as phonophoresis with sodium diclofenac gel as coupling agent does have a significant effect on the symptoms of tennis elbow, both on subjective and objective symptoms. The result shows that in the treatment of tennis elbow, low level laser therapy was no better than phonophoresis approach. It may be possible that larger samples would have enabled to reveal a significantly greater improvement in favor of phonophoresis group, since there was a tendency towards betterness in Phonophoresis group.

In a study by Joseph A Balogun et al, for analysis of the gender main effect, it was found that in both sexes (male \& female) significant differences exist when grip strength was tested in different postures and elbow joint angles. The result of their study revealed that males had higher grip strength than females at any posture and any elbow joint angles ${ }^{22}$. In this study for all testing conditions, significant positive correlation was obtained between grip strength and age. The current study groups turned out well balanced in respect of both the sex and age so the chance of bias by age and gender is less in this study.

In the current study, statistically significant improvement in pain, as measured by the VAS, FPS, Pain free grip strength was present in the group received phonophoresis therapy. Therefore present study provides information which may be useful when considering the use of sodium diclofenac preparation as couplants during ultrasound treatment of soft tissue injuries and musculoskeletal conditions. As per Burnham R et al Topical diclofenac appears to provide effective short-term reduction in elbow pain and wrist extensor weakness associated with chronic lateral epicondylitis. The outermost layer of the epidermis or the stratum corneum is recognized as the primary barrier to the transdermal penetration of medications and physical agents .Factors that alter the integrity of this layer can dramatically affect diffusion across $i t^{11}$. Therapeutic ultrasound irradiation enhances the percutaneous penetration of topical diclofenac ge ${ }^{12}$. For the mechanisms by which US acts as an enhancer of drug delivery both the thermal and non-thermal properties of US have been considered. Cavitation induced lipid bilayer disordering was found to be the most important cause for ultrasonic enhancement of transdermal transport ${ }^{21}$. Theoretically, the use of a sodium diclofenac coupling medium in the treatment of tennis elbow is an attractive concept. The proximity of the lateral epicondyle to the skin would favor the use of this method, because the ultrasonically driven sodium diclofenac should have a shorter distance to travel to its site of action.

In the current study, statistically significant improvement in pain, as measured by the VAS, FPS and Pain free grip strength was present in the group received laser therapy. Different studies have used different types of low intensity laser for pain relief, lasers of $904 \mathrm{~nm}$ wavelength were found to be effective on the treatment of tendonitis ${ }^{14,15,16}$. The photostimulation effect of LLLT on tissues induces a cascade of signaling events initiated by the initial absorption of light by cytochrome oxidase. These signaling events may include the activation of immediate early genes transcription factors, cytochrome oxidase subunit gene expression, and a host of other enzymed and pathways related to increased oxidative metabolism ${ }^{23}$. LLLT has been reported to suppress inflammation by a reduction of $\mathrm{PGE}_{2}$ in ligament cell cultures ${ }^{24}$. This study showed that laser had similar healing powers on the patients with lateral epicondylitis as shown by a significant reduction of symptoms in the elbow and it is also showed laser therapy to be no more effective than phonophoresis therapy with diclofenac as a coupling agent.

Because the ultra structural characteristics, vascularity, and biomechanical demands of the involved tissues are quite different, a uniform response to treatment should not be assumed ${ }^{10}$. This may explain why few patients had 
poor response to either of the treatments. Repetitive activity, especially house wife often precipitate the onset and failure to rest from this activity resulted in poor response. In the current study it was given only individual modalities as interventions without any other combinations of therapy and advices. M ost of the patients in this study were of working class and could not rest the involved part throughout the treatment session, which could have hampered the expected outcome.

One of the interesting findings of this study was the response to treatment demonstrated by the acute patients. This finding was also observed in another study by Lesley $\&$ David $^{25}$. Of the ten patients referred for treatment within three months of onset of symptoms, 8 patients (80\%) reported gaining benefit from treatment. The majority of patients (75\%), presented with symptoms greater than 3 months duration together with a multitude of failed previous treatments. The opportunity to study a large group of acute cases with minimal previous treatment was therefore denied. There is very little, reported in the literature about the possible importance of early referral of patients. These findings confirm the need for further studies of large groups of acute cases.

VAS, FPS evaluation methods had some limitations although these methods are well documented. The methods include a highly subjective component which reflects the patient's individual pain behavior or pain thresholds and have an effect on the results. In addition, pain free grip strength may be affected by the patient's illness behavior. In clinical practice these kinds of evaluation methods are widely used. Therefore, from a clinical viewpoint, the results and methods of this study can be considered as acceptable. It may be also possible to have a strong halo effect where the first scale dominates the readings obtained from successive scales. This has important consequences since it is common to assess several subjective phenomena using a series of rating scales within a single interview. The results of the present investigation do not unfortunately clarify this issue.

It is believed that lateral epicondylitis has a tendency towards spontaneous healing. This process can take anything from 12 to 18 months. Cyriax regarded tennis elbow as a self-limiting condition, improving with or without treatment within 8-12 months. Unfortunately, there are no published reports that address the issue of the rate of spontaneous recovery. In this study there was no control group, therefore comparisons with the control group cannot be made with full certainty and the conclusions must be interpreted with caution. Further, as the control group received no treatment some improvement in the two treatment groups could be attributed to placebo effect and supervision. Inclusion of a placebo group would be helpful. Ambiguities regarding spontaneous recovery can be resolved only by monitoring the course of the disease more closely over a more prolonged period of at least one year and introducing control groups.

Limitations of the study could be, since most of the patients in this study were of working class, it was difficult for them to give adequate rest for the involved part throughout the course of treatment, this could have hampered the expected outcome and in this study there was no control group, therefore comparisons with the control group cannot be made with full certainty. Still there is lack of literature regarding at what stage in the disease process the modalities should be used The result of the present study do not fortunately clear this issue. In future studies, we feel that for the accurate evaluation of physiotherapy technique in the treatment of lateral epicondylitis these features should be included; Trails should consist of large numbers of patients referred for treatment as early as possible preferably within three months from onset of symptoms, Previous treatments should be taken into consideration, Placebo treatments should be used to compare the effects of various modalities and close objective and subjective monitoring should be maintained for a minimum period of one year.

\section{Conclusion :}

This study concludes that low level laser therapy and 
Phonophoresis with sodium diclofenac gel as coupling agent gives a good result in the treatment of lateral epicondylitis. This study also concludes that, statistically there was no significant difference was found between Low level laser therapy and Phonophoresis with sodium diclofenac gel as coupling agent in the management of lateral epicondylitis.

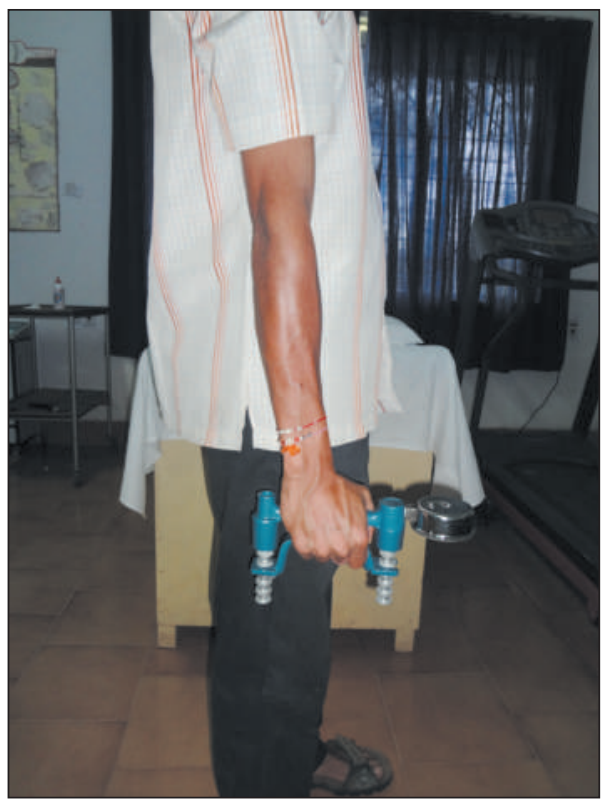

Figure 1: Pain free grip strength measurement with Jamar Hand Dynamometer

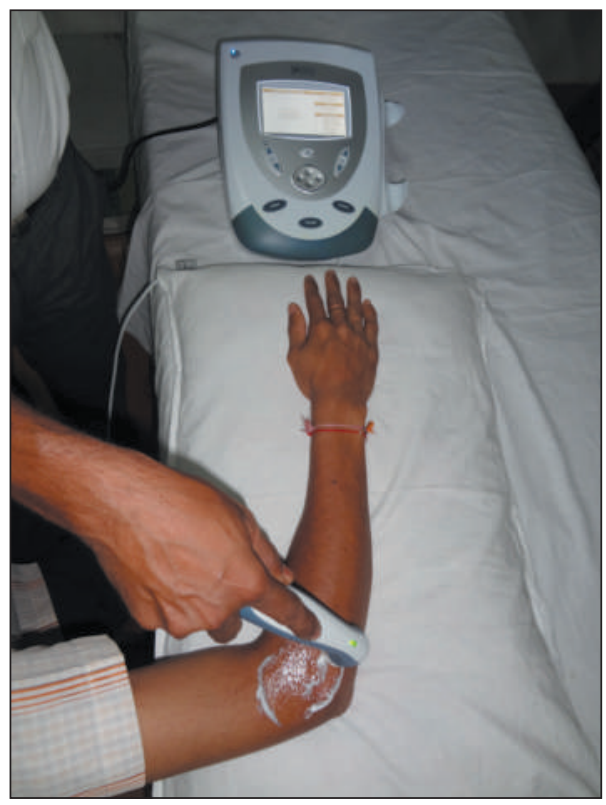

Figure 3: M ethod of application of Phonophoresis

\section{Acknowledgements:}

Authors would like to thank the Department of Orthopaedics of KS Hegde Hospital and the participants for their support.

\section{Conflict of Interest :}

Authors agree that there was no source of conflict of interest.

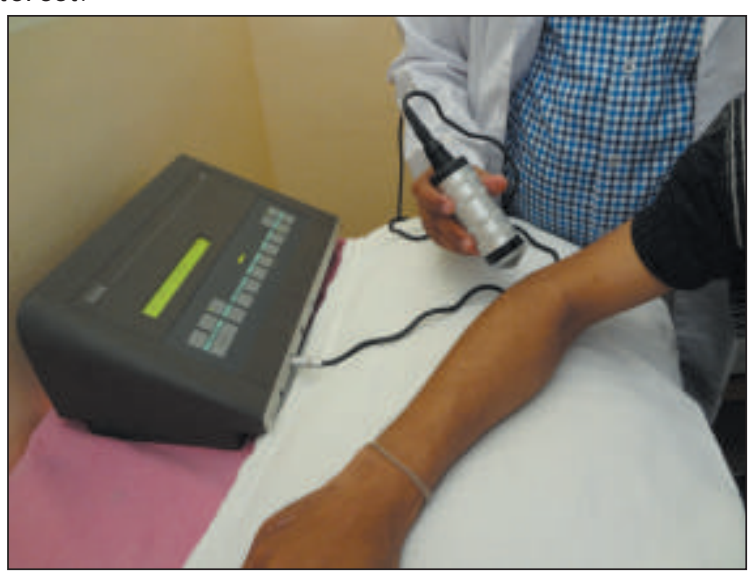

Figure 2: M ethod of application of Low Level Laser Therapy

Table 1: Age distribution

Table 1: Age distribution

\section{Group Statistics}

\begin{tabular}{|cc|cc|c|c|}
\hline & GROUP & $\mathrm{N}$ & Mean & Std. Deviation & $\mathrm{Z}$ \\
\hline AGE & Group A & 20 & 34.9000 & 6.6878 & 1900 \\
\cline { 3 - 6 } & Group B & 20 & 33.8000 & 10.1442 & $\mathrm{P}=849 \mathrm{~ns}$ \\
\hline
\end{tabular}

Table 2: Sex Distribution

Sex distribution

\begin{tabular}{|ccc|c|c|c|}
\hline \multirow{2}{*}{} & & \multicolumn{2}{|c|}{ GROUP } & \multirow{2}{*}{ Total } \\
\cline { 3 - 5 } & & Group A & Group B & \\
\hline SEX & $\mathrm{M}$ & Count & 12 & 11 & 23 \\
& & $\%$ & $60.0 \%$ & $55.0 \%$ & $57.5 \%$ \\
\hline & $\mathrm{F}$ & Count & 8 & 9 & 17 \\
& & $\%$ & $40.0 \%$ & $45.0 \%$ & $42.5 \%$ \\
\hline Total & & Count & 20 & 20 & 40 \\
& & $100.0 \%$ & $100.0 \%$ & $100.0 \%$ \\
\hline
\end{tabular}

Table: 3: Dominant and non dominant side comparison SIDE

\begin{tabular}{|c|c|c|c|c|}
\hline & \multicolumn{2}{|l|}{ GROUP } & \multirow[b]{2}{*}{ Total } \\
\hline & & Group A & Group B & \\
\hline \multirow[t]{2}{*}{ Dominant } & Count & 17 & 17 & 34 \\
\hline & $\%$ & $85.0 \%$ & $85.0 \%$ & $85.0 \%$ \\
\hline \multirow{2}{*}{$\begin{array}{l}\text { Non } \\
\text { Dominant }\end{array}$} & Count & 3 & 3 & 6 \\
\hline & $\%$ & $15.0 \%$ & $15.0 \%$ & $15.0 \%$ \\
\hline Total & Count & 20 & 20 & 40 \\
\hline
\end{tabular}


Table: 4: Group statistics of VAS, FPS and Pain free grip strength for group $A$ and group $B$ before treatment

Group Statistics

\begin{tabular}{|ll|c|c|c|c|}
\hline & GROUP & $\mathrm{N}$ & Mean & Std. & $\mathrm{Z}$ \\
\hline VASPR & Group & 20 & 5.9000 & 1.4614 & .7800 \\
& Group & 20 & 5.7000 & 1.1098 & $\mathrm{P}=.435 \mathrm{~ns}$ \\
\hline FPSPR & Group & 20 & 23.6000 & 3.9523 & .8160 \\
& Group & 20 & 24.6000 & 3.6332 & $\mathrm{P}=.414 \mathrm{~ns}$ \\
\hline Pain free & Group & 20 & 13.9500 & 5.3653 & .9290 \\
Strength-Pre & Group & 20 & 14.4000 & 3.5748 & $\mathrm{P}=.353 \mathrm{~ns}$ \\
\hline
\end{tabular}

Table: 5: The paired difference of pre to post scores for VAS, FPS and Pain free grip strength in group $A$ and Group $B$.

\section{PRE TO POST}

\begin{tabular}{|lc|c|c|c|c|}
\hline \multirow{2}{*}{ GROUP } & \multicolumn{2}{|c|}{ Paired Differences } & & \\
\cline { 3 - 5 } & & Mean & Std. Deviation & $\mathrm{Z}$ & $\mathrm{p}$ \\
\hline Group A VAS & 3.3500 & 1.9042 & 3.853 & .001 vhs \\
& FPS & 11.6000 & 7.1259 & 3.928 & .001 vhs \\
Pain free grip strength & -5.5500 & 4.6957 & 3.744 & .001 vhs \\
\hline Group A VAS & 3.1000 & 1.4510 & 3.928 & .001 vhs \\
& FPS & 8.3500 & 3.5285 & 3.934 & .001 vhs \\
Pain free grip strength & -4.7000 & 3.4504 & 3.841 & .001 vhs \\
\hline
\end{tabular}

Table: 6: Comparison of differences from pre to post between groups

\begin{tabular}{|lc|c|c|c|c|}
\hline & GROUP & N & Mean & Std. Deviation & Z \\
\hline VAS & Group & 20 & 3.3500 & 1.9042 & .6270 \\
& Group & 20 & 3.1000 & 14510 & $p=53 \mathrm{~ns}$ \\
\hline FPS & Group & 20 & 11.6000 & 7.1259 & 1.1430 \\
& Group & 20 & 8.3500 & 3.5285 & $\mathrm{p}=.253 \mathrm{~ns}$ \\
\hline Pain free & Group & 20 & -5.5500 & 4.6957 & .7950 \\
grip strength Group & 20 & -4.7000 & 3.4504 & $\mathrm{p}=.426 \mathrm{~ns}$ \\
\hline
\end{tabular}

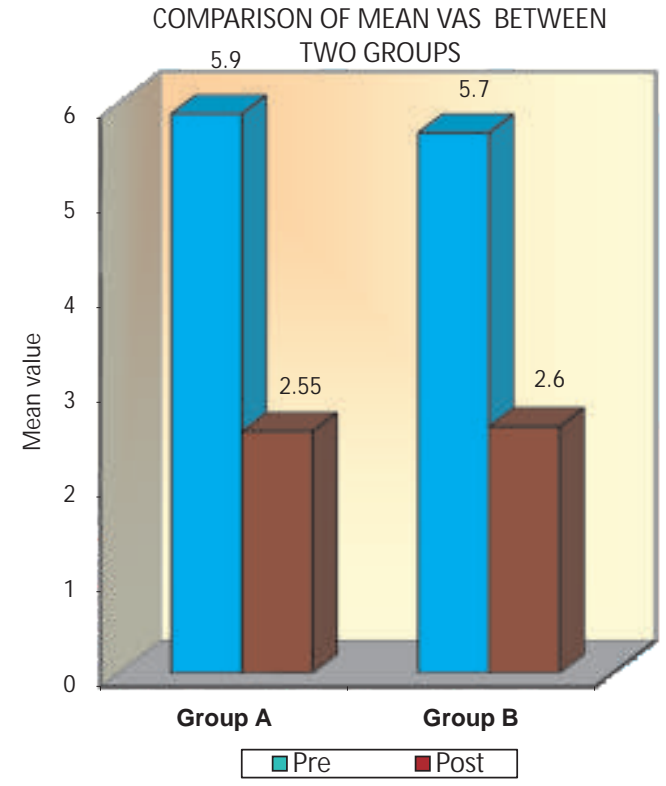

Graph 1: Comparison of mean VAS between 2 groups

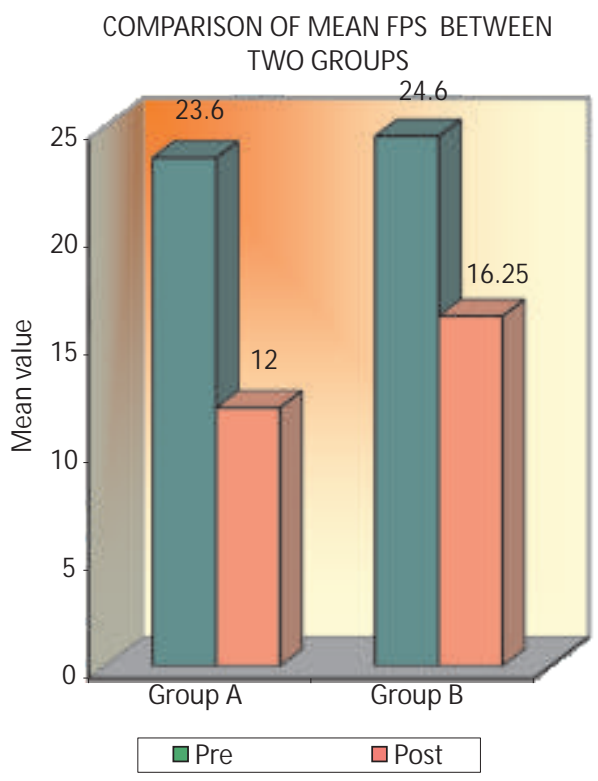

Graph 2: Comparison of mean FPS between 2 groups

COMPARISON OF MEAN PAIN FREE GRIP STRENGTH BETWEEN TWO GROUPS

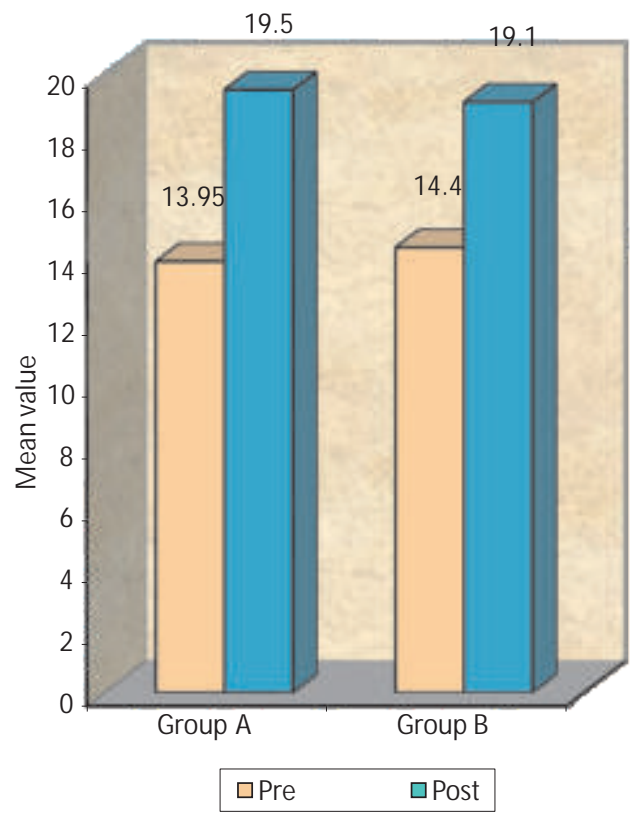

Graph 3 : Comparison of mean pain free grip strength between 2 groups

References:

1. Tuomo T Pienimaki, Tuula K Tarvainen, Pertti $T$ Siira, Heikki Vanharanta. Progressive strengthening and stretching exercises and ultrasound for chronic lateral epicondylitis. Physiotherapy. 1996. September; 82 (9): 522 - 530.

2. William E GarrettJr., Kevin P. Speer and Donald T. Kirkendall. Principles and Practice of Orthopaedic Sports M edicine. Philadelphia: Lippincott WilliamsP Wilkins; 2000.

3. James Cyriax. Text book of Orthopaedic M edicine. $6^{\text {th }}$ edition. London: Tailliere Tindall \& Casstel; 1975.

4. Thomas G Wadsworth. Tennis elbow: Conservative, Surgical, and M anipulative treatment. British M edical Journal 1987 M arch;294: 621 $-624$. 
5. M aria Zuluaga, Christopher Briggs, Jhon Carlisle,Virginia Mc Donald, Joan Mc Meeken, Wendy Nickson. Sports physiotherapy; Applied science and practice. $1^{\text {st }}$ Edition. Hong Kong: Churchill Livingstone; 1995.

6. Monica Kesson and Elanie Atkins. Orthopaedic Medicine: A practical Approach. Great Britian: Butterworth \& Heinemann; 1998.

7. David C. Reid. Sports Injury Assessment and Rehabilitation. United States of America: Churchill Livingstone; 1992.

8. Alain - Yvan Belanger. Evidence - Based guide to therapeutic physical agents. United States of America: Lippincott Williams and Wilkins; 2002.

9. John Low and Ann Reed. Electrotherapy Explained - principles and practice. ${ }^{\text {rd }}$ Edition. India: Butterworth \& Heinemann; 2001.

10. Mark D. Klaiman, Joseph A. Shrader, Jerome V Danoff, Jeanne E. Hicks, William J. Pesce and James Ferland. Phonophoresis versus ultrasound in the treatment of common musculoskeletal conditions. Medicine and Science in Sports and Exercise 1998 M ay; 1349 - 1355.

11. Burnham R, Gregg R Healy P, Steadward. The effectiveness of topical Diclofenac for lateral epicondylitis. Clinical Journal Sports M edicine. 1998. pril; 8 (2): 78-81.

12. Rosim GC, Barbieri CH ,Lancas F M,Mazzer N. Diclofenac phonophoresis in human volunteers. Ultrasound Medical Biology. $2005 \mathrm{March}$; 3(3): 337 - 43.

13. Audrey Lowe, Jean Wessel, M ichele C Battie. Test - Retest Reliability, Construct Validity, and Responsiveness of a Functional Pain Scale for Tennis Elbow. Physiotherapy Canada 2003; 55(2): 114 - 122.

14. Ottar Vasseljen Jr, Nils Hoeg, Berit Kjeldstad, Anders J ohnsson and Stig Larsen. Low level laser versus placebo in the treatment of Tennis elbow. Scandinavian Journal of Rehabilitation M edicine 1992;24:37-42.

15. Haker E, Lundeberg T. Is low - energy laser treatment effective in lateral epicondylalgia? Journal Pain Symptom Management 1991 May; $6(4): 241-6$.
16. Tam G. Low power Laser therapy and analgesic action. Journal Clinical Laser M edical Surgery 1999 February; 17 (1): 29 - 33.

17. Heather A E Benson, James C M CElnay. Topical Non - steroidal Anti inflammatory products as ultrasound Couplants: Their potential in Phonophoresis. Physiotherapy 1994 February; 80 (2): 74 - 76.

18. A Binder, G Hodge, A M Greenwood, BL Hazleman, D P Page Thomas. Is therapeutic ultrasound effective in treating soft tissue lesions? British Medical Journal February 1985; 290: 512 - 514.

19. M oneet Kochar, Ankit Dogra. Effectiveness of a specific physiotherapy regimen on patient's with Tennis Elbow. Physiotherapy 2002 June; 88 (6): $333-341$

20. Ottar Vasseljen. Low-Level Laser versus Traditional Physiotherapy in the treatment of Tennis Elbow. Physiotherapy 1992 May; 78(5):329334

21. Samir Mitragotri, David A. Edwards, Daniel Blankschtein, Robert Langer. A M echanistic study of Ultrasonically- Enhanced Transdermal Drug Delivery. Journal of Pharmaceutical sciences1995; 84(6):697706

22.Joseph A Balogun, Charles T Akomolafe, Lateef 0 Amusa. Grip strength: Effects of testing posture and elbow position. Archives physical medical Rehabilitation 1991April; 72:280-283.

23. Dr. Harry Whelan. Thor Laser therapy for wound Healing. Physical and occupational Therapy in Geriatrics 2000; 18(2): 1-19.

24. Jan M Bjordal , Christian Couppe, Roberta TChow ,Jan Tuner, Elisabeth Anne Ljunggren .A systemic review of low level laser therapy with location specific-doses for pain from chronic joint disorders. Australian Journal of Physiotherapy 2003; 49:107-115.

25. Leslley K Holdsworth, David M Anderson. Effectiveness of ultrasound used with a Hydrocortisone coupling medium or epicondylitis clasp to treat lateral epicondylitis: pilot study. Physiotherapy 1993 January; 79(1):19-25 